\title{
Drinking Behavior Among College Students: Interventions to Increase Mindfulness and Social Capital
}

\author{
Jenna C. Anderson, MS, CHES \\ Mississippi State University, Starkville, Mississippi, United States
}

Antonio J. Gardner, PhD, CHES

Mississippi State University, Starkville, Mississippi, United States

(iD) https://orcid.org/0000-0002-9721-0244

Barry P. Hunt, EdD

Mississippi State University, Starkville, Mississippi, United States

Contact: ajg130@msstate.edu

\section{Abstract}

The impacts of drinking behaviors are far-reaching, with college student drinking contributing to over 1,500 deaths and an estimated 696,000 assaults on college campuses each year. There are a variety of explanations for why college students engage in risky drinking behaviors. However, evidence suggests that drinking and other substance use may be a result of complex psychological origins, including adverse childhood experiences (ACEs). This paper reviews the literature available on ACEs among college students, how these experiences impact alcohol use among this population, and potential areas for intervention, including those with a focus on mindfulness and social capital.

Keywords: alcohol, college, adverse childhood experiences, mindfulness, social capital

Date Submitted: August 11, 2020 | Date Published: February 23, 2021

\section{Recommended Citation}

Anderson, J. C., Gardner, A. J., \& Hunt, B. P. (2021). Drinking behavior among college students: Interventions to increase mindfulness and social capital. Journal of Social, Behavioral, and Health Sciences, 15, 76-87.

https://doi.org/10.5590/JSBHS.2021.15.1.05

\section{Introduction}

It is well known that campus substance misuse, abuse, and addiction is a serious, widespread problem accounting for many deaths, physical and sexual assaults, and damage to on- and off-campus property (National Institute on Alcohol Abuse and Alcoholism [NIAAA], 2019). Evidence suggests that drinking and other substance use may be a result of complex psychological origins, including adverse childhood experiences (ACEs; Smyth et al., 2008). Students who have previously experienced ACEs are more likely to experience distress, mental health issues, and make poor choices about substance use (Hinojosa et al., 2019).

Note: This research was not supported by any grants.

Correspondence concerning this article should be sent to Antonio J. Gardner, PhD, MS, CHES, Department of Food Science, Nutrition, \& Health Promotion, Mississippi State University, Mailstop 9805, Starkville, MS 39762. 
The purpose of this paper was to review current literature on ACEs and vulnerability to alcohol abuse in college students, with specific emphasis on two cognitive deficits that often accompany young adults who have experienced ACE: emotional dysregulation and experiential avoidance (Tripp et al., 2015). The focus then turned to the efficacy of mindfulness-based interventions and social capital interventions. A discussion of the merits and potential of each approach is followed by implementation considerations, recommendations for assessment, and future research directions.

\section{Alcohol Abuse on Campus}

College students and high rates of alcohol use are almost synonymous. The 2017 National Survey on Drug Use and Health documented alcohol use among college students aged 18-22, reporting that $53.6 \%$ of full-time college students reported drinking alcohol in the past month and $34.8 \%$ said they participated in binge drinking, while 9.7\% admitted to heavy alcohol use (NIAAA, 2019). The impacts of these drinking behaviors are far-reaching, with college student drinking contributing to over 1,500 student deaths and an estimated 696,000 assaults by students who have been drinking on college campuses each year (NIAAA, 2019).

\section{Adverse Childhood Experiences (ACEs)}

Research has suggested that exposure to ACEs could be a motivating factor behind alcohol and other substance use and abuse among college students (Flood et al., 2009; Forster et al. 2017; Read et al., 2012; Tripp et al., 2015; Windle et al., 2018). According to Karatekin (2018), ACEs are "moderately to severely stressful experiences during the first 18 years of life, including emotional, sexual, and physical abuse, neglect, parental psychopathology, parental incarceration, and parental separation and/or divorce" (p. 36). ACEs among college students are quite common (Smyth et al., 2008). Results from three prevalence studies surveying over 6,000 college students from varying colleges, college types, and geographical regions, indicated that $55.8 \%$ to $84.5 \%$ of college students had experienced at least one ACE in their lives thus far (Smyth et al., 2008). Karatekin (2018) found that 64\% of students at a large midwestern university had experienced at least one ACE, that having had one ACE increased the likelihood of experiencing multiple events, and that effects are often cumulative.

As a result of previous exposure to ACEs, some college students meet the criteria for a diagnosis of posttraumatic stress disorder (PTSD; Tripp et al., 2015). In fact, prevalence studies indicated that $6-12 \%$ of college students with a history of past trauma meet the criteria for an official PTSD diagnosis (Tripp et al., 2015). However, while some students develop PTSD or other diagnosable psychological conditions as a result of exposure to adverse experiences, not everyone exposed to ACEs develops a psychological disorder (Read et al., 2012). According to Read et al. (2012), "a substantial portion of trauma-exposed individuals do not meet full criteria for PTSD diagnosis, but experience significant trauma-associated distress" (p. 426). Students with symptoms fewer than the standard for PTSD had similar numbers of substance-related consequences as those with the official diagnosis (Read et al., 2012). While not all students with past ACEs develop PTSD or other diagnosable mental health conditions, they are still more likely to have experienced "traumatic stress" at some point, which is "both acute and chronic and is characterized by intense feelings of horror, fear, or helplessness that overwhelm normal coping mechanisms" (Hinojosa et al., 2019, p. 531). This is important to understand since many students may be overlooked for potential interventions designed to address underlying psychological issues if only those who have been identified as having diagnosed psychological conditions are targeted. 


\section{Impact of ACEs}

According to Windle et al. (2018), ACEs have been associated with a variety of unhealthy behavior choices among adolescents and young adults, including higher levels of substance use, cigarette use, and obesity. Hinojosa et al. (2019) asserted that college students who have been exposed to ACEs were both more likely to experience mental health issues such as depression and/or suicidality, as well as partake in risky behaviors such as risky sexual activity, eating disorders, and alcohol and other drug use. Windle et al. (2018), summarized previously, conducted research in the field suggesting that those who have been exposed to ACEs may not have had the emotional and physical protection they needed during their early development and thus may be unable to protect themselves or evaluate risks of certain behaviors.

Evidence suggests that ACEs can cause biological changes in people that can alter how they respond to perceived stressful situations in everyday life (Karatekin, 2018; Windle et al., 2018; Woolman et al., 2015). Windle et al. (2018) pointed out that actual alterations in hormonal and immune system factors can take place as a result of trauma, which can lead to the restructuring of an individual's brain, making them more susceptible to feeling stressed. Particularly significant levels of stress during a person's development can leave them more vulnerable to feeling the sensation of stress not just as a child, but long into the future (Karatekin, 2018). This sensitivity can increase perceptions of stress, including those commonly found among college students, such as stressors related to academics, or even exacerbate already impactful symptoms of PTSD (Woolman et al., 2015).

\section{ACEs and Substance Use}

For students who experience ACEs and develop diagnosable psychological conditions and those who do not, research suggests that they are likely at higher risk of alcohol and/or substance abuse (Flood et al., 2009; Forster et al., 2017; Read et al., 2012; Tripp et al., 2015; Windle et al., 2018). Individuals who suffer from symptoms of PTSD often suffer from substance abuse disorders (Flood et al., 2009; Tripp et al., 2015).

Research conducted on the relationship between ACEs and substance use among college students has shown a strong association between the number of ACEs experienced and the amount of substance use (Forster et al., 2017), with one study finding that higher ACE scores were significant predictors of increased substance use (Windle et al., 2018).

Research suggested that college students exposed to ACEs were at a higher risk for substance use because alcohol and other substances were used as common coping mechanisms to deal with and/or escape psychological distress (Flood et al., 2009; Roche et al., 2019; Tripp et al., 2015; Tubbs et al., 2019; Woolman et al., 2015). Alcohol and other substances were often used as forms of "self-medication," which soothes otherwise negative emotional states (Flood et al., 2009; Read et al., 2012; Tripp et al., 2015). This was often referred to as the "self-medication hypothesis," which stated that substance use happens because of an attempt to try to tamper distressing feelings, symptoms, or memories surrounding a trauma (Read et al., 2012, p. 427).

Common themes in the literature suggested that a lack of emotion regulation skills (Roche et al., 2019; Tripp et al., 2015) and high experiential avoidance (Roche et al., 2019; Tubbs et al., 2019) could be the driving factors behind the relationship between psychological distress as a result of ACEs and the use and/or abuse of alcohol and other substances among college students.

Emotion regulation is "an ability to refrain from impulsive behavior when experiencing negative emotions, acceptance of emotions, access to emotion regulation strategies perceived as effective, and understanding of emotions" (Tripp et al., 2015, p. 108). Individuals who do not have appropriate levels of emotion regulation skills were likely to be more impulsive and utilize strategies that provide immediate relief from difficult emotions, even if those strategies were unhealthy (Roche et al., 2019). 
Experiential avoidance is an "unwillingness to be in contact with internal experiences (such as thoughts, emotions, memories, urges, or bodily sensations) and any attempt to alter, change, or control such experiences" (Roche et al., 2019, p. 18). Those who have experienced trauma in their life may be especially likely to utilize experiential avoidance techniques in order to avoid painful memories or intrusive thoughts so that they may experience immediate, short-term relief (Roche et al., 2019). Flood et al. (2009) studied the connection between PTSD and negative health outcomes among college students and found that use of alcohol and other drugs was associated with students with PTSD, which researchers suggested was likely a result of attempts to self-medicate and regulate emotions that were difficult for them to manage. Although selfmedication may provide short-term relief for students who are experiencing difficult emotions, avoidance and suppression of negative emotions and thoughts subsequently may lead to the worsening of psychological distress (Tubbs et al., 2019).

Young adults entering college may be particularly at risk for unhealthy self-medication techniques because emerging adulthood is a common time for the advent of a variety of psychological disorders (Miller-Graff et al., 2015). In fact, $75 \%$ of many mental health disorders develop by the age of 24 (Karatekin, 2018). Mental health conditions among young adults in America are increasing, which is also increasing the risk for challenges in relation to academics, dropout rates, violence, injury, and suicide (Miller-Graff et al., 2015). The first year of college often comes with an increased sense of autonomy and feelings of instability, along with an increase in substance use among college students (Read et al., 2012). Interventions at this stage could prove to be a much-needed, as well as effective, way to ensure that students with exposure to ACEs do not turn to unhealthy forms of self-medication.

\section{Choosing an Intervention}

\section{Mindfulness}

Level of mindfulness is a factor that may be influential in determining the frequency of problematic behaviors among college students in general (Barrington et al., 2019), as well as among those with adverse childhood experiences or otherwise difficult childhoods (Brett et al., 2018; Roche et al., 2019).

Mindfulness interventions are potential ways to assist college students in dealing with a variety of stressors (Dvořáková et al., 2017; Felver et al., 2018; Vinci et al., 2014). Mindfulness is a "nonreactive, nonjudgmental awareness of thoughts, emotions, behaviors and sensations in the present moment" (Brett et al., 2018, p. 93). It is a technique that can be used to manage all sorts of emotions, including negative ones, and has five frequently researched components consisting of observing, describing, acting with awareness, non-judgment, and non-reactivity (Roche et al., 2019). The observing component includes looking at and observing the present moment; describing is being able to describe personal experiences in the present moment; acting with awareness is being aware of personal thoughts, feelings, and actions in the present moment instead of being on "autopilot"; non-judgment is seeing an inner experience for what it is and not placing judgment on it; and non-reactivity is accepting inner experience and emotions as they are and not being quick to react to them (Roche et al., 2019). Examples of what these components of mindfulness may look like in real-world application can be seen through analysis of items used in the Five Facet Mindfulness Questionnaire (FFMQ), which Roche et al. (2019) used in their study to document mindfulness practices of college students.

Various interventions designed to improve mindfulness skills have been shown to be effective in reducing psychological distress (Felver et al., 2018) and issues with depression, anxiety, and sleep (Dvořáková et al., 2017), as well as in improving overall stress, resilience, and self-efficacy levels (Vidic \& Cherup, 2019) among college students. As discussed previously, many college students may partake in experiential avoidance, or avoiding negative emotions, as a way of coping. Also, a popular way of coping with and/or avoiding negative emotions is by using substances such as alcohol. Another related concept is that of negative affect. Negative 
affect, in summary, is characterized by negative emotional states associated with life experiences such as anger, guilt, fear, and nervousness (Vinci et al., 2014).

According to Vinci et al. (2014), mindfulness could be a viable way to help individuals understand and accept their emotions instead of avoiding them, which is important since it is thought that the presence of negative affect can greatly influence substance use behaviors. In their study, Enríquez et al. (2017) found that a mindfulness program implemented among college students had significant impacts on study participants, in that students were more able to put their emotional experiences into perspective, problem solve how to resolve issues, and be able to see positives in otherwise negative experiences.

\section{Mindfulness and Problematic Behaviors}

Literature on mindfulness suggests that mindfulness and alcohol consumption may be negatively correlated (Barrington et al., 2019; Vinci et al., 2014). Barrington et al. (2019) pointed out several studies showing that higher levels of mindfulness components, such as acting with awareness, non-judging, and describing, were associated with lower levels of alcohol consumption. Evidence indicated that being exposed to ACEs may lead to deficits in mindfulness skills as a result of poor memory, reduced self-regulation, and higher likelihood of difficult-to-manage emotions, which may then lead to alcohol consumption as a way of coping (Brett et al., 2018, p. 93).

Evidence also shows that this relationship between mindfulness and alcohol use is present in the college student population (Barrington et al., 2019; Brett et al., 2018; Roche et al., 2019). In a study conducted on the relationship between dispositional mindfulness and problematic alcohol and cannabis use among college students in general, results indicated that students with higher levels of mindfulness typically had lower levels of alcohol and cannabis use (Barrington et al., 2019). A study done on the relationship between adverse childhood experiences, mindfulness, and various health-related behaviors among college students found that mindfulness impacted the relationship between adverse childhood experiences and alcohol use and consequences in the study sample (Brett et al., 2018). In particular, results suggest that components of mindfulness, such as acting with awareness and non-judgment, may be among the most important components influencing use of alcohol and associated consequences, with lower levels of acting with awareness and non-judgment associated with students who had past experience with ACEs drinking more (Brett et al., 2018). Research also indicates that two components of mindfulness, acting with awareness and non-judgment of inner experiences, mediated the relationship between childhood trauma and problem behaviors such as excessive alcohol use, sexual promiscuity, and nicotine use (Roche et al., 2019).

\section{Mindfulness Interventions}

Based on assessment information gathered directly from a target population of college students, there should be an indication of what kind of mindfulness intervention or combinations of interventions would be appropriate for a specific campus. For example, factors such as whether participation in the intervention should be voluntary or mandatory (Dvořáková et al., 2017), whether it will be for-credit or not (Vidic \& Cherup, 2019), and if these should be in-person at certain identified times or available online for students to access as they please (Oehme et al., 2019) should be considered. Mindfulness training or classes could easily be introduced to college students through new student orientation classes, resident hall programs, or simply as voluntary opportunities available to students should they desire to participate (Dvořáková et al., 2017).

When making this decision, it may be efficacious to explore mindfulness interventions that could be used for course credit or mandatory academic requirements (Dvořáková et al., 2017; Vidic \& Cherup, 2019). Vidic and Cherup (2019) recommended consideration of mindfulness interventions being integrated into students' academic curriculum as a requirement or option for academic credit. This approach was seen as more proactive, in that students will receive information and develop skills related to mindfulness while fulfilling 
other obligations that may help alleviate psychological issues as they are occurring, instead of after major issues develop (Vidic \& Cherup, 2019).

When deciding on a particular program to use inside or outside of classrooms, administers should consider programs that emphasize acting with awareness among participants, especially when there is a desire to reduce rates of problematic drinking through the development of mindfulness skills (Barrington et al., 2019; Roche et al., 2019; Vinci et al., 2014). Research shows that acting with awareness is negatively associated with problematic drinking among college students (Vinci et al., 2014).

\section{Social Capital}

In the literature on problem behaviors among youths and adolescents, the concept of social capital appears prominently (Curran, 2007; Kotch et al., 2014; Magson et al., 2016; Seon et al., 2019). Social capital is "a person's social networks and relationships that promote healthy development” (Seon et al., 2019, p. 23). Individuals may find social capital in their families, social groups, schools, and communities; it can take emotional, physical, or material form; and is, overall, the social networks that people have, including the benefits they receive as a result of having those social networks (Seon et al., 2019). Essentially, someone with a high level of social capital has strong social relationships with others around them to the point that they gain valuable life insight and skills through their interaction with others.

\section{Social Capital and Problematic Behavior}

The levels of social capital among youths and problem behaviors appear to be related (Curran, 2007; Kotch et al., 2014; Magson et al., 2016). In a study conducted on the relationship between social capital and substance use among high school students, researchers found that the social relationships adolescents have with their families, peers, schools, and communities greatly influenced the level of alcohol, tobacco, marijuana, and other drug use (Curran, 2007).

This relationship can also be seen among youth with particularly difficult childhoods (Kotch et al., 2014; Magson et al., 2016). In a study on the impact of social capital, isolation, and a sense of belonging on the frequency of risk-taking behaviors among disadvantaged youth, adolescents presented with fewer risk-taking behaviors when they had higher levels of social capital and a sense of belonging (Magson et al., 2016). Community social capital was found to be most predictive of reduced youth risk-taking behavior (Magson et al., 2016). An association was also found between higher levels of peer social capital and less alcohol use (Magson et al., 2016). In an analysis conducted on a longitudinal study called LONGSCAN, which follows children through time with experiences of child maltreatment, Kotch et al. (2014) examined the potential impact of child maltreatment on later externalization of behavioral problems and indicated that adolescents with a history of neglect are much more likely to have externalizing behavioral problems such as alcohol use and smoking.

Based on the literature concerning the relationship between social capital and problematic behaviors among youth, individuals with lower levels of social capital available to them growing up appeared more likely to not receive the social and emotional support needed to develop self-regulating emotional and decision-making skills, thus making them more vulnerable to problematic behaviors. Using the aforementioned definition of ACEs as a reference, it would also appear that individuals with past exposure to ACEs may lack useful levels of social capital, as experience with ACEs often means experience with unhealthy social interactions, including various forms of abuse at the hands of those in their own social networks (Karatekin, 2018). It can reasonably be suggested that this relationship likely also applies to the college student population. 


\section{Social Capital Interventions}

As previously established, social capital has been identified as a potential factor that influences the health and well-being of youth with traumatic or negative childhoods (Kotch et al., 2014; Magson et al., 2016; Seon et al., 2019) through influencing factors such as academic achievement (Seon et al., 2019) and development of problematic behaviors (Kotch et al., 2014; Magson et al., 2016). Seon et al. (2019) asserted that both formal and informal support were important for the academic success of college students with adverse backgrounds, especially when they first arrive on campus. Also, formal supports such as those from professionals working in the counseling center and health center appeared to be particularly important in the success of college students with unstable childhoods (Seon et al., 2019).

Accordingly, the establishment of formal support networks for college students upon arrival appears to be important so that students feel that they have the emotional, informational, and material support needed to address academic, emotional, and physical needs. Seon et al. (2019) suggested that a relationship between students and formal support services be established through efforts expelled during the development of freshman orientation practices, residence hall programs, or the involvement of these departments in any supports designed to address the needs of students with unstable childhoods. Interventions such as the LIFE program (Hurtado et al., 2019) hold promise as systematic, institutional-level interventions designed to enhance student connectedness with a variety of these social support resources. Informal support networks are also necessary. These include relationships between students and their peers, which can and should be developed through the use of informal gatherings, study groups, or student orientation groups (Seon et al., 2019).

\section{Screening for ACEs}

Recently, there has been a steady increase in demand for mental health services among college students, but these concerns are typically not met with sufficient attention or resources on campuses (Karatekin, 2018; Miller-Graff et al., 2015). This supply and demand issue often leads to a narrow focus on crisis intervention and short-term patches to chronic problems that may need consistent attention (Miller-Graff et al., 2015).

To try and ensure that students who are at the highest potential risk for mental health issues and related risky behaviors are supported, many researchers suggest figuring out ways to identify students with a history of trauma exposure and/or trauma-related mental health conditions when they enter college (Forster et al., 2017; Hinojosa et al., 2019; Karatekin, 2018; Miller-Graff et al., 2015). Some researchers believe that campuswide screenings could be used for this identification process (Forster et al., 2017; Hinojosa et al., 2019; Karatekin, 2018; Smyth et al., 2008; Woolman et al., 2015). This could be done through screening for previous exposure to violence (Miller-Graff et al., 2015), ACEs (Forster et al., 2017; Karatekin, 2018), traumarelated disorders (Hinojosa et al., 2019), or by adding assessment tools that assess previous trauma exposure and its resulting implications for alcohol screenings and interventions (Woolman et al., 2015).

Administers should consider the potential ethical issues associated with screening individuals for adverse childhood experiences (Finkelhor, 2018). With the variety in types of ACEs and their associated impacts on people, it can prove difficult to ensure that appropriate interventions are given to individuals with exposure to ACEs once they are identified (Finkelhor, 2018). There also is the potential of psychological distress among those being screened as a result of accompanying stigma and worry, as well as the potential for false positives and over diagnosis, which can lead to wasted resources (Finkelhor, 2018).

As a result of concerns such as these, perhaps screening should not automatically be considered as necessary on each college campus, but instead that mindfulness and social capital interventions be available to all students, not just those students with ACEs. It appears appropriate to design and implement mental health 
interventions that can improve the lives and health-related choices of all students. Interventions and approaches with an emphasis on mindfulness skills and gains in social capital may be particularly useful in the reduction of problematic substance use among the college population.

\section{Recommendations for Assessment/Implementation}

Levels of mindfulness and social capital may have the potential to influence the frequency of problematic behaviors, including alcohol use. However, before jumping into the selection of any one type of intervention, universities should conduct appropriate assessments, such as determining what kinds of attitudes and beliefs students have surrounding the use of alcohol. This assessment should primarily be done through direct interaction with students. It is important to get their opinion about how problematic drinking behaviors are on their campus, if they feel they or others would benefit from interventions geared toward this issue, what factors influence why they drink, and their thoughts on potential interventions geared toward this topic. This would be a good time to introduce potential thoughts on the influence of social networks in relation to social capital and mindfulness skills to see if students think these could be good points of intervention. Focus groups, forums, individual interviews, or surveys would be useful at this time.

It is also essential to understand the kinds of health issues college students are facing on a given campus and what specific factors are contributing to rates of specific illnesses and problematic behaviors. One could look at statistics of college students in general, similar to what is presented in this paper, as well as existing statistics available on various campuses in relation to how common alcohol use is, frequency of alcoholrelated illnesses and/or mental health issues, and alcohol-related consequences faced by students. Such data could be found through coordination with departments such as Health Services, Counseling Services, and/or Campus Safety, as well as through evaluation of available national college health data. This may be a time to conduct anonymous gathering of data about the percentage of students who report having exposure to adverse childhood experiences or otherwise traumatic pasts, as well as how these events may impact their current lifestyle and/or academic ability.

Proper assessment will need to also include evaluation of available resources on campus that could be used to conduct a particular health-related intervention. This evaluation should focus on resources needed to reduce problematic drinking behavior, including but not limited to, mindfulness- and social capital-related interventions. Resources to consider are available funding, manpower, expertise, technology, materials, and administrative approval (McKenzie et al., 2017). This may be achieved through the creation of a coalition of essential individuals in and around the campus community, which would allow for the sharing of information about available resources needed for a chosen intervention.

The creation of a coalition of members of most, if not all, departments present on campus would be of benefit when establishing what course of action should be taken to influence drinking behaviors of students, as well as deciding if an approach focused on mindfulness and/or social capital would be appropriate and feasible. Coalitions allow for the sharing of essential resources, as mentioned above. Suggested members of this coalition would include, but are not limited to, campus administrators, counseling services, health services, professors, the division of student affairs, peer health groups and peer health educators, residence life and housing, chaplains, campus safety, sports coaches, family members, and, of course, the students. It may also be efficacious to determine what resources are available off campus in the surrounding community, such as additional mental health providers, health educators, or other health professionals.

In summary, interventions that seek to reduce maladaptive coping strategies among college students in response to stress and negative affect should consider the influence of exposure to ACEs among college students, acknowledge the importance of appropriate assessment, utilize partnerships and collaboration 
among a variety of entities, work to increase levels of social capital through enhancement of student social support, utilize mindfulness skill building in order to reduce stress, and encourage emotional self-regulation.

If assessment of the available literature on mindfulness and social capital and an appropriate needs assessment on individual campuses indicate that a mindfulness and/or social capital approach to problematic drinking could be beneficial, it may be efficacious to implement interventions that focus on these key areas. We recommend that a program based on needs assessments, conducted to determine appropriateness for each college campus population and collaboration among key stakeholders, be used to promote positive coping mechanisms among college students. Attempts to screen students for ACEs should be approached with caution, especially if not anonymous. Use of a variety of approaches to address health-related issues and provide a plethora of avenues for students to develop knowledge and health-related skills may encourage more interest among students. Appropriate assessment should allow for an idea of what would be best received on each college campus, but, based on the literature, interventions with a focus on mindfulness and social capital should be considered.

\section{Conclusion}

College students partake in problematic behaviors, including drinking for a variety of reasons, but the negative influence of adverse childhood experiences and resulting development of maladaptive coping mechanisms may, at least in part, explain this relationship. According to the literature, factors including levels of mindfulness and social capital may play a part in moderating the relationship between college students with ACEs and problematic behavior. Because of this, interventions geared toward reducing problematic drinking behaviors among college students may be successful if there is a focus on increasing mindfulness and social capital on college campuses. Therefore, we recommend a multidimensional approach that attempts to positively influence both factors through the collaboration of interested stakeholders and appropriate assessment of student needs and desires. 


\section{References}

Barrington, J., Weaver, A., \& Brebner, K. (2019). Exploring mindfulness in relation to alcohol and cannabis use among first year university students. College Student Journal, 53(2), 163-174.

Brett, E. I., Espeleta, H. C., Lopez, S. V., Leavens, E. L. S., \& Leffingwell, T. R. (2018). Mindfulness as a mediator of the association between adverse childhood experiences and alcohol use and consequences. Addictive Behaviors, 84, 92-98. https://doi.org/10.1016/j.addbeh.2018.04.002

Curran, E. M. (2007). The relationship between social capital and substance use by high school students. Journal of Alcohol \& Drug Education, 51(2), 59-73.

Dvořáková, K., Kishida, M., Li, J., Elavsky, S., Broderick, P. C., Agrusti, M. R., \& Greenberg, M. T. (2017). Promoting healthy transition to college through mindfulness training with first-year college students: Pilot randomized controlled trial. Journal of American College Health, 65(4), 259-267. https://doi.org/10.1080/07448481.2017.1278605

Enríquez, H., Ramos, N., \& Esparza, O. (2017). Impact of the Mindful Emotional Intelligence Program on emotional regulation in college students. International Journal of Psychology \& Psychological Therapy, 17(1), 39-48.

Felver, J. C., Morton, M. L., \& Clawson, A. J. (2018). Mindfulness-based stress reduction reduces psychological distress in college students. College Student Journal, 52(3), 291-298.

Finkelhor, D. (2018). Screening for adverse childhood experiences (ACEs): Cautions and suggestions. Child Abuse \& Neglect, 85, 174-179. https://doi.org/10.1016/j.chiabu.2017.07.016

Flood, A. M., McDevitt-Murphy, M. E., Weathers, F. W., Eakin, D. E., \& Benson, T. A. (2009). Substance use behaviors as a mediator between posttraumatic stress disorder and physical health in trauma-exposed college students. Journal of Behavioral Medicine, 32(3), 234-243. https://doi.org/10.1007/s10865008-9195-y

Forster, M., Grigsby, T. J., Rogers, C. J., \& Benjamin, S. M. (2017). The relationship between family-based adverse childhood experiences and substance use behaviors among a diverse sample of college students. Addictive Behaviors, 72, 298-304. https://doi.org/10.1016/j.addbeh.2017.08.037

Hinojosa, R., Nguyen, J., Sellers, K., \& Elassar, H. (2019). Barriers to college success among college students that experienced adverse childhood events. Journal of American College Health, 67(6), 531-540. https://doi.org/10.1080/07448481.2018.1498851

Hurtado, I., Hunt, B. P., \& Gardner, A. J. (2019). Enhancing student success with a connectedness focused retention initiative: The LIFE Program. Journal of Student Success and Retention, 6(1), 63-72.

Karatekin, C. (2018). Adverse childhood experiences (ACEs), stress, and mental health in college students. Stress \& Health: Journal of the International Society for the Investigation of Stress, 34(1), 36-45. https://doi.org/10.1002/smi.2761

Kotch, J. B., Smith, J., Margolis, B., Black, M. M., English, D., Thompson, R., Lee, L., Taneja, G., \& Bangdiwali, S. I. (2014). Does social capital protect against the adverse behavioral outcomes of child neglect? Child Abuse Review, 23(4), 246-261. https://doi.org/10.1002/car.2345

Magson, N. R., Craven, R. G., Munns, G., \& Yeung, A. S. (2016). It is risky business: Can social capital reduce risk-taking behaviours among disadvantaged youth? Journal of Youth Studies, 19(5), 569-592. https://doi.org/10.1080/13676261.2015.1098776

McKenzie, J. F., Neiger, B. L., \& Thackeray, R. (2017). Planning, implementing, \& evaluating health promotion programs: A primer (7th ed.). Pearson Benjamin Cummings. 
Miller-Graff, L. E., Howell, K. H., Martinez-Torteya, C., \& Hunter, E. C. (2015). Typologies of childhood exposure to violence: Associations with college student mental health. Journal of American College Health, 63(8), 539-549. https://doi.org/10.1080/07448481.2015.1057145

National Institute on Alcohol Abuse and Alcoholism. (2019). Fall semester: A time for parents to discuss the risks of college drinking. https://www.niaaa.nih.gov/publications/brochures-and-fact-sheets/timefor-parents-discuss-risks-college-drinking

Oehme, K., Perko, A., Clark, J., Ray, E. C., Arpan, L., \& Bradley, L. (2019). A trauma-informed approach to building college students' resilience. Journal of Evidence-Based Social Work, 16(1), 93-107. https://doi.org/10.1080/23761407.2018.1533503

Read, J. P., Colder, C. R., Merrill, J. E., Ouimette, P., White, J., \& Swartout, A. (2012). Trauma and posttraumatic stress symptoms predict alcohol and other drug consequence trajectories in the first year of college. Journal of Consulting \& Clinical Psychology, 8o(3), 426-439. https://doi.org/10.1037/aoo28210

Roche, A. I., Kroska, E. B., Miller, M. L., Kroska, S. K., \& O’Hara, M. W. (2019). Childhood trauma and problem behavior: Examining the mediating roles of experiential avoidance and mindfulness processes. Journal of American College Health, 67(1), 17-26. https://doi.org/10.1080/07448481.2018.1455689

Seon, J., Prock, K. A., Bishop, J. D., Hughes, A. K., Woodward, A. T., \& MacLean, M. (2019). Formal and informal social support and academic achievement among college students with unstable childhood experiences. Child Welfare, 97(1), 21-43.

Smyth, J. M., Hockemeyer, J. R., Heron, K. E., Wonderlich, S. A., \& Pennebaker, J. W. (2008). Prevalence, type, disclosure, and severity of adverse life events in college students. Journal of American College Health, 57(1), 69-76. https://doi.org/10.3200/JACH.57.1.69-76

Tripp, J. C., McDevitt-Murphy, M. E., Avery, M. L., \& Bracken, K. L. (2015). PTSD symptoms, emotion dysregulation, and alcohol-related consequences among college students with a trauma history. Journal of Dual Diagnosis, 11(2), 107-117. https://doi.org/10.1080/15504263.2015.1025013

Tubbs, J. D., Savage, J. E., Adkins, A. E., Amstadter, A. B., \& Dick, D. M. (2019). Mindfulness moderates the relation between trauma and anxiety symptoms in college students. Journal of American College Health, 67(3), 235-245. https://doi.org/10.1080/07448481.2018.1477782

Vidic, Z., \& Cherup, N. (2019). Mindfulness in classroom: Effect of a mindfulness-based relaxation class on college students' stress, resilience, self-efficacy and perfectionism. College Student Journal, 53(1), 130-142.

Vinci, C., Peltier, M. R., Shah, S., Kinsaul, J., Waldo, K., McVay, M. A., \& Copeland, A. L. (2014). Effects of a brief mindfulness intervention on negative affect and urge to drink among college student drinkers. Behaviour Research \& Therapy, 59, 82-93. https://doi.org/10.1016/j.brat.2014.05.012

Windle, M., Haardörfer, R., Getachew, B., Shah, J., Payne, J., Pillai, D., \& Berg, C. J. (2018). A multivariate analysis of adverse childhood experiences and health behaviors and outcomes among college students. Journal of American College Health, 66(4), 246-251. https://doi.org/10.1080/07448481.2018.1431892

Woolman, E. O., Becker, M. M., \& Klanecky, A. K. (2015). PTSD symptoms mediate academic stress and drinking to cope in college students. Journal of Drug Education, 45(2), 96-112. https://doi.org/10.1177/0047237915607282 
Anderson et al., 2021

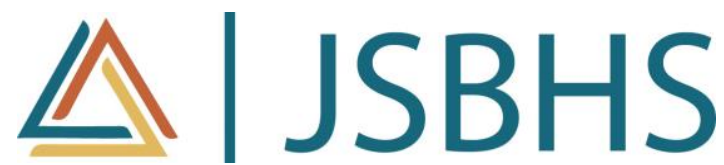

The Journal of Social, Behavioral, and Health Sciences (JSBHS), co-sponsored by the College of Health Sciences and the College of Social and Behavioral Sciences at Walden University, is a peer-reviewed, online, interdisciplinary journal focusing on theoretically-based research that addresses contemporary national and international issues. $J S B H S$ articles include peer-reviewed research reports, brief reports, comprehensive literature reviews, books reviews, and student research. 